\title{
ARTICLE Relative quantification of phosphoproteomic changes in grapevine (Vitis vinifera L.) leaves in response to abscisic acid
}

\author{
Supakan Rattanakan ${ }^{1}$, Iniga George ${ }^{2}$, Paul A Haynes ${ }^{2}$ and Grant R Cramer ${ }^{1}$
}

In a previous transcriptomic analysis, abscisic acid ( $A B A)$ was found to affect the abundance of a number of transcripts in leaves of Cabernet Sauvignon grapevines with roots that had been exposed to $10 \mu \mathrm{M} A B A$ for $2 \mathrm{~h}$. Other work has indicated that ABA affects protein abundance and protein phosphorylation as well. In this study we investigated changes in protein abundance and phosphorylation of Cabernet Sauvignon grapevine leaves. Protein abundance was assessed by both label-free and isobaric-label quantitive proteomic methods. Each identified common proteins, but also additional proteins not found with the other method. Overall, several thousand proteins were identified and several hundred were quantified. In addition, hundreds of phosphoproteins were identified. Tens of proteins were found to be affected in the leaf after the roots had been exposed to ABA for $2 \mathrm{~h}$, more than half of them were phosphorylated proteins. Many phosphosites were confirmed and several new ones were identified. ABA increased the abundance of some proteins, but the majority of the proteins had their protein abundance decreased. Many of these proteins were involved in growth and plant organ development, including proteins involved in protein synthesis, photosynthesis, sugar and amino-acid metabolism. This study provides new insights into how ABA regulates plant responses and acclimation to water deficits.

Horticulture Research (2016) 3, 16029; doi:10.1038/hortres.2016.29; Published online 22 June 2016

\section{INTRODUCTION}

Grapevines (Vitis vinifera L.) are an economically important fruit crop worldwide. They are used for the production of wine, table grapes, juice and raisins, and are worth millions of dollars every year for the US industry. Abiotic stresses affect both quality and quantity of grape production. ${ }^{1,2}$ Mild drought stress or the application of abscisic acid (ABA) increase phenolic compounds such as anthocyanin, catechin and quercetin in the fruit $^{3-5}$ and, in part because of their antioxidant activities, can benefit human health. Severe water deficit can reduce photosynthesis, inhibit vine growth, and decrease the quality of grapevines. ${ }^{6}$ Thus, there is an optimal level of drought stress that produces an optimal grape wine quality. A better understanding of grapevines responses to drought stress will allow one to minimize the loss of grapevine production and maximize grape quality.

ABA is a plant hormone that has important roles in developmental processes and adaptive stress responses in plants such as salt, cold and drought stress. ${ }^{7,8}$ ABA regulates plant responses by altering protein activities directly by posttranslational modifications such as phosphorylation and nitrosylation, and indirectly by affecting the transcription of many genes. ${ }^{9-11}$ A model of ABA signaling has been constructed and involves a central core pathway of PYR/PYL/RCAR receptors, 2C-type protein phosphatases (PP2C) and SNF1-related protein kinase 2 (SnRK2). ${ }^{12,13}$ Several transcription factors (AREB/ ABFs) and ion channel proteins (SLAC1 and KAT1) are phosphorylated by SnRK2 kinases, $^{14,15}$ but very likely there are many more proteins to be identified. Moreover, there may be other kinases in the $A B A$ signaling pathway that have yet to be discovered. ${ }^{10,11}$

Omic technologies have been used to gain better understanding of plant responses to stresses. ${ }^{16}$ Despite the great advances transcriptomic analyses have contributed to our understanding, there are far fewer proteomic and phosphoproteomic studies, which address a different level of plant regulation. Furthermore, recent studies from our lab indicate that the abundance of most proteins is not well correlated with transcript abundance. ${ }^{17,18}$

In a previous study, the transcriptomic responses of grapevine to $A B A$ were examined. ${ }^{19}$ Some of the results from that study indicated that the roots, which had been treated with $1 \mu \mathrm{M} \mathrm{ABA}$ for $2 \mathrm{~h}$ had 538 significantly differentially expressed genes (DEGs), whereas the leaf from the same plant had 69 significantly DEGs in response to the root treatment. Genes with significantly increased transcript abundance in leaves were involved in protein folding and the protein amino-acid phosphorylation process in roots. In this paper, we extend this study by examining the proteomic and phosphoproteomic responses of the grapevine leaves of vines whose roots were treated with ABA.

In this study, we identify proteins and phosphoproteins involved in the ABA signaling pathway in grapevine. A label-free approach was first used to identify and quantify changes in protein abundance. In addition, we utilized a second approach, using 6-plex isobaric mass tagging technology, labeling peptides with structurally identical tags but different reporter ions. Our data sets revealed motifs and phosphorylation sites that are consistent with other plant phosphoproteomes. ${ }^{11,20-24}$ 


\section{MATERIALS AND METHODS}

Sample collection and ABA treatment

Rooted cuttings of Cabernet Sauvignon grapevines were grown in a growth chamber for 2 to 3 weeks before carefully transferring them to an aeroponic system located in a greenhouse under standard conditions (with supplemental sodium vapor lamp lighting ( $16 \mathrm{~h}$ light (minimum $400 \mu \mathrm{E} \mathrm{m}^{-2} \mathrm{~s}^{-1}$ ) at $28^{\circ} \mathrm{C}$ and $8 \mathrm{~h}$ dark at $18^{\circ} \mathrm{C}$ cycle). Each container $(43.2 \mathrm{~cm}(L) \times 27.9 \mathrm{~cm}(M) \times 20.3 \mathrm{~cm}(H))$ had a nebulizer with a fogger head size of $3.8 \mathrm{~cm}$ diameter $\times 4.4 \mathrm{~cm}$ height for each experimental replicate (three containers for control and three containers for ABA treatment). The lid of each container had small holes large enough for several rooted plants to be passed through and into the container. Gibeaut's solution ${ }^{25}$ was used to provide the macronutrients and micronutrients to the vines. The $\mathrm{pH}$ of the solution was maintained at 6.0. Root and leaf samples were grown for 3 months before treatment.

ABA was applied to the roots by continually misting the roots with $10 \mu \mathrm{m}$ $A B A$ added to the Gibeaut's solution in the aeroponic system; leaves from the same vine (but not directly exposed to the misting solution) and exposed roots were collected after $2 \mathrm{~h}$ of root exposure with fresh control and $A B A$ solutions. Root samples were quickly rinsed with tap water and collected leaf and root materials were rapidly frozen in liquid nitrogen before storage at $-80^{\circ} \mathrm{C}$.

\section{Label-free approach}

Proteome sample preparation and LC-MS/MS analysis. A phenol extraction protocol was used for Vitis vinifera leaves and is based on previous protocols (Vincent, Wheatley et al. 2006). ${ }^{17,18}$ Trypsin in-solution digestion, peptide extraction and fraction analysis by nanoflow liquid chromatography tandem mass spectrometry (LC-MS/MS) were conducted essentially as previously described. ${ }^{26}$ Briefly, three experimental replicates of ABA-treated leaves and untreated leaves were run separately on an LTQ Velos Pro mass spectrometer (Thermo, San Jose, CA, USA) for the sampleoptimized gas phase fractionation. Chromatography was performed on an Easy-nLC II (Thermo) with magic C18 AQ column ( $3 \mu \mathrm{m}$ bead size, $200 \AA$ pore size, $0.1 \mathrm{~mm}$ inside diameter $\times 100 \mathrm{~mm}$; Michrom Biosciences, Auburn, CA, USA). Each sample was analyzed in four 120-min LC-MS/MS runs at $0.5 \mu \mathrm{L} \mathrm{min}{ }^{-1}$, each using a different precursor mass range $(400-$ $506,501-658,653-913$ and $908-1600$ ). The $\mathrm{m} / \mathrm{z}$ ranges for four gas phase fractions per sample were optimized empirically by analyzing a mixture of pooled samples from $m / z 400-1600$, then creating gas phase fractionation fractions to approximately evenly distribute peptide observations among the four fractions.

Protein identification and quantification. A protein database for $V$. vinifera was combined as in a previous report (Cramer et al. $\left.{ }^{18}\right)$. The X! Tandem and the GPM Cyclone (www.thegpm.org) in the automated mode using MudPIT merging were used for peptide-to-spectrum matching. Protein and peptide false discovery rates (FDR) were calculated using reverse database searching. ${ }^{27}$

Low-stringency protein identification data from individual replicate experiments were transformed into high-stringency data sets containing only reproducibly identified proteins using a suite of $\mathrm{R}$ modules known as the Spectral Counting Reporting Analysis Program (Scrappy). ${ }^{28}$ For a protein to be considered as reproducibly identified it needed to satisfy two criteria: it must be present with at least five peptides across all three replicates, an average of 1.7 peptides per experiment. The same program was used for calculation of protein abundance using normalized spectral abundance factors (NSAF), and determination of significantly differentially expressed proteins $(P<0.05)$ based on Student's $t$-test across three replicate NSAF values.

\section{TMT labeling approach}

Proteome sample preparation. The phenol protein extraction was used as mentioned above. The protein concentration was determined by using EZQ protein quantitation according to the manufacturer's instructions (Bio-Rad, Hercules, CA, USA). Protein samples $(10 \mathrm{mg})$ were digested with trypsin and desalted by Sep Pak Plus C18 cartridges (Waters Inc, Milford, MA, USA). Peptides (10 mg) were resuspended in $100 \mu \mathrm{L}$ of $1 \%$ acetic acid.

Phosphopeptides enrichment and TMT labeling. The immobilized metal affinity chromatography (IMAC column; Qiagen Ni-NTA spin column (Cat No. 31014)) was loaded with $50 \mu \mathrm{L}$ of peptides. The IMAC column was washed four times; twice with 1 bead volume of $0.1 \mathrm{~m} \mathrm{NaCl} / 25 \% \mathrm{ACN} /$
$0.1 \%$ acetic acid, once with 1 bead volume of $1 \%$ acetic acid, and once with half bead volume of $\mathrm{ddH}_{2} \mathrm{O}$. Peptides were eluted with $3 \times$ bead volume using $6 \% \mathrm{NH}_{4} \mathrm{OH}$ (total $120 \mu \mathrm{L}$ ); the elution was collected in a silanized glass insert, and then dried with a speed-vac. The tandem mass tags (TMT) labeling method was followed according to the manufacturer's instructions (TMT Fisher \# 90061, Thermo Fisher Scientific, Asheville, NC, USA). The untreated leaf samples were labeled with TMT-126, TMT-127, and TMT-128, while ABA-treated leaf samples were labeled with TMT-129, TMT-130, and TMT-131. All TMT labeling samples were combined before sample fractionation using hydrophilic interaction liquid chromatography according to Pucci et al. (Pucci, Giuliano et al. 2009). A total of 40 fractions were evaporated to dryness in a vacuum centrifuge and resuspended in $100 \mu \mathrm{L}$ $5 \%$ CAN and $0.1 \%$ formic acid for LC-MS/MS analysis.

LC-MS/MS analysis. Digested peptide samples were analyzed using LC-MS/MS at the Nevada Proteomics Center (University of Nevada, Reno, NV, USA). The peptides were separated and analyzed using a Michrom Paradigm Multi-Dimensional Liquid Chromatography instrument (Michrom Bioresources Inc., Auburn, CA, USA) coupled with a Thermo LTQ Orbitrap $\mathrm{XL}$ mass spectrometer (Thermo Fisher Scientific, San Jose, CA, USA). Peptide samples were dissolved in $100 \mu \mathrm{L}$ of $0.1 \%$ formic acid and loaded onto a ZORBAX 300SB- $\mathrm{C}_{18} 5-\mu \mathrm{m}(5 \times 0.3 \mathrm{~mm})$ trap column (Agilent Technologies, Santa Clara, CA, USA), eluted from the trap, and then separated with a reverse phase Michrom Magic $C_{18} A Q$ column $(3 \mu \mathrm{m}$, $200 \AA, 0.2 \times 150 \mathrm{~mm})$ by a gradient elution using solvent $\mathrm{A}(0.1 \%$ formic acid) and solvent $B(0.1 \%$ formic acid in $\mathrm{ACN})$ at a flow rate of $2 \mu \mathrm{L} \mathrm{min}{ }^{-1}$. The gradient was set from 5 to $40 \%$ solvent B for 90 min, increased to $80 \%$ solvent B in $10 \mathrm{~s}$ and held at $80 \%$ solvent B for $1 \mathrm{~min}$. MS spectra were recorded over the mass range of $m / z 400-1600$ with resolution of 60000 . The three most intense ions were isolated for fragmentation in the linear ion trap using CID with minimal signal of 500 and collision energy of 35.0 or using HCD with a minimal signal of 1000 , collision energy of 55.0, and an activation time of $30 \mathrm{~ms}$. Dynamic exclusion was implemented with two repeat counts, repeat duration of $15 \mathrm{~s}$ and exclusion duration of $90 \mathrm{~s}$.

Protein identification and quantification. All MS/MS samples were analyzed using Sequest (Thermo Fisher Scientific; version 1.0). Sequest was set up to search the uniprot_Vitis_20121010 database (54 242 entries). Search parameters included fragment ion mass tolerance of $1.00 \mathrm{Da}$ and a parent ion tolerance of 10.0 p.p.m., trypsin enzyme specificity, carbamidomethylation of cysteine as a fixed modification and oxidation of methionine, phosphorylation of serine, threonine and tyrosine and TMT6plex of lysine and the $\mathrm{N}$-terminus as variable modifications. Scaffold (version Scaffold_4.4.1, Proteome Software Inc., Portland, OR, USA) was used to validate MS/MS based peptide and protein identifications. Peptide identifications were accepted if they could be established at greater than $95.0 \%$ probability by the Peptide Prophet algorithm ${ }^{29}$ with Scaffold delta-mass correction. Protein identifications were accepted if they could be established at $>95.0 \%$ probability and contained at least 1 identified peptide.

Relative abundances of proteins were calculated based on Dayon et $a l^{30}$ Briefly, a normalization of the reporter intensities by the sum of all the reporter intensities was made in order to determine the relative abundance of each reporter. Then the $\log _{2}$ ratio of average TMT $129,130,131$ / $\mathrm{TMT}^{126,127,128}$ (ABA/Control) were obtained from the average values. Finally, an unpaired $t$-test was assessed to test the significance of the abundance difference $(P<0.0 .5)$.

\section{Motif analysis}

In order to identify potential enzyme recognition sites, Scaffold PTM scans the data set for over-represented patterns in the amino acids surrounding modification sites. It uses the iterative statistical method described in a previous publication. ${ }^{31}$ Sequence logos were generated using Weblogo ${ }^{32}$ of all phosphorylation sites with Ascores $\geqslant 13(P<0.05)$. The frequency of each residue present in each data set is proportional to its height. The phosphorylation site is located at 0 on the $x$ axis flanking by 12 amino-acid residues (at position -6 to +6 ).

\section{RESULTS}

Proteomic changes in leaves of grapevine in response to $A B A$ Leaves of Cabernet Sauvignon grapevines were collected after $2 \mathrm{~h}$ treatment with $10 \mu \mathrm{M}$ ABA to the roots of the same vines. In this study, two approaches were employed to identify and quantify 
proteomic changes (Figure 1). The first method was label-free quantitative shotgun proteomics using nanoflow liquid chromatography-tandem mass spectrometry (nanoLC-MS/MS). The second method was isobaric chemical labeling using TMT. The 6-plex TMTs were used to label leaf control $\left(\right.$ TMT $\left.^{126,127,128}\right)$ and ABAtreated samples $\left(\right.$ TMT $\left.^{129,130,131}\right)$ after phosphopeptide enrichment by IMAC. Protein identification and quantitation were analyzed after LC-MS/MS analysis.

Label-free quantitative proteomic analysis of grapevine leaves in response to $A B A$ was quantified by normalized spectral abundance factors. ${ }^{28}$ Approximately 2533 non-redundant Vitis vinifera proteins in the UniProtKB database were identified at low stringency, with 363 and 310 proteins reproducibly identified in samples of leaf control and leaf ABA, respectively, at a FDR of $0.55 \%$ (Table 1 ; Supplementary File 1 ). Among the total of identified proteins, 20 upregulated and 13 downregulated proteins were significantly differentially expressed in the leaves in response to ABA treatment of the roots $(P<0.05)$ (Table 2$)$. A functional analysis for Gene Ontology (GO) categories was analyzed with the Cytoscape (3.2.0, www.cytoscape.org) and the BinGO plugin (3.0.3, www.cytoscape.org) for the statistically significant proteins in response to ABA (Supplementary File 2), using a custom annotation derived from UniProt (uniprot.org), EnsemblPlants (plants.ensembl.org) and Gramene (gramene. org). ${ }^{33,34}$ Photosynthesis, carbohydrate catabolic process and response to abiotic stimulus were significantly over-represented GO categories after correcting for FDR (adjusted $P$ value of 0.05 ) for both significant down- and upregulated proteins in response to ABA. Serine family amino-acid metabolic process was

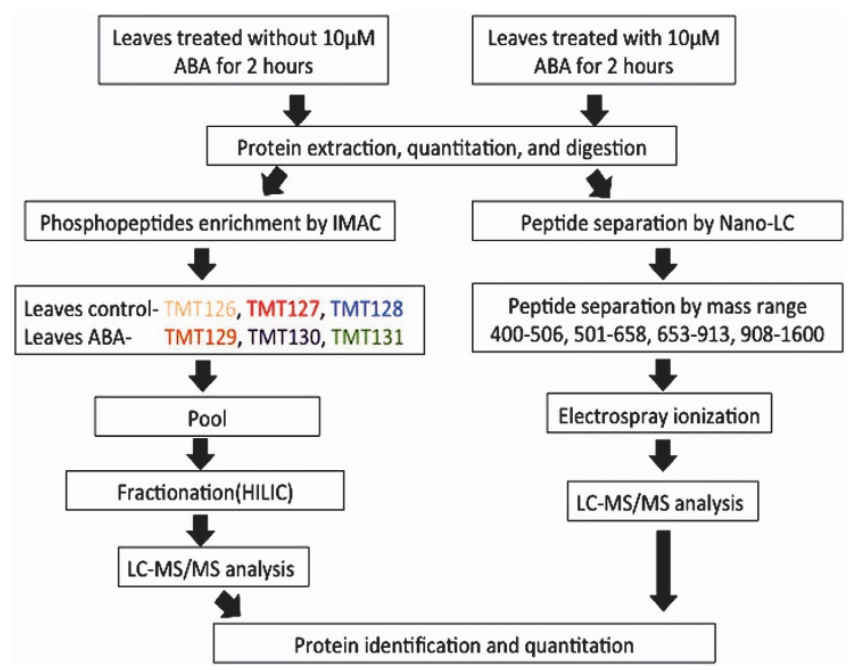

Figure 1. A workflow of a comprehensive large-scale MS-based proteomics and phosphoproteomics strategy. MS, mass spectrometry. significantly over-represented in downregulated proteins (F6HTU8, F6HTS6, D7SQ37 and D7TAY3), whereas protein folding was significantly over-represented only in upregulated proteins (F6HLR2, D7SJX8 and F6HDM4).

Some of the proteins decreased in abundance by ABA include photosynthetically related proteins such as a photosystem I complex protein (D7TAY3) and a oxygen-evolving enhancer 3 protein, PsbQ (F6H8B4), part of photosystem II. A ribosomal protein (F6HSE3), involved in protein synthesis, was also decreased. Other proteins affected by ABA include proteins involved in amino acid, sugar and cell wall metabolism. A few proteins were increased in protein abundance by $A B A$ including a voltage-dependent anion channel (A5AUG8), an NADP-dependent malic enzyme (P51615) and a putative oxygen-enhancer protein (Q6XGX7).

A total of 1011 proteins were identified by TMT labeling in the leaves of grapevine in response to $10 \mu \mathrm{M} A B A$ for $2 \mathrm{~h}$ at an FDR of $0.62 \%$ at the protein level and $0.9 \%$ at the peptide level. (Supplementary File 3). A total of 787 proteins were tagged with TMT on the free amino terminus or lysine residues with a $0.61 \%$ protein FDR and $0.8 \%$ peptide FDR (Supplementary File 4). It should be noted that all proteins reported in this table with quantitation data have been observed in triplicates of both conditions, allowing for statistical evaluation of quantitation differences.

There were 20 proteins ( 11 phosphoproteins) that were significantly changed in protein abundance $(P \leqslant 0.05)$ in the leaves in response to the roots being treated with $A B A$ (Table 3). All significant differentially expressed proteins in response to $A B A$ decreased in protein abundance. Biological process analysis (GO) of significant proteins changing in protein abundance in response to $A B A$ revealed that several developmental processes were affected ( $P \leqslant 0.05$; Supplementary File 5$)$. These proteins include the acetyltransferase component of the pyruvate dehydrogenase complex (D7TZW9), a Nck-associated protein 1 (D7T9L3) involved in actin remodeling, a component (F6HTW0) of the Cul4-RING E3 ubiquitin ligase complex, a DNA/RNA helicase (F6GT26), a protein involved in stability of Photosystem II (F6HVA4), a eukaryotic translation initiation factor (F6I2I6) and a ribosomal protein (A5Al30).

\section{ABA affects phosphoproteins in grapevine}

We utilized a new approach for the identification and quantification of phosphoproteins by enrichment of phosphopeptides prior to TMT labeling. This approach gives valuable data on phosphoprotein identification, phosphorylation sites and protein abundance changes. There were 219 phosphoproteins identified by TMT-LC-MS/MS (Supplementary File 6) and 116 of them were tagged with TMT. The phosphoproteins in this table are sorted by the number of discrete peptides identified; 145 proteins were identified from multiple peptides and 74 proteins were identified from single peptides. However, these proteins are not subject to the usual concerns associated with single peptide-based protein identifications because they were actually identified and quantified in triplicate experiments to allow for statistical evaluation.

Table 1. Peptide/protein identification data of Cabernet Sauvignon leaves: control and ABA

\begin{tabular}{|c|c|c|c|c|c|c|c|c|}
\hline \multirow[t]{2}{*}{ Condition } & \multicolumn{3}{|c|}{$\begin{array}{c}\text { Low stringency redundant } \\
\text { peptide count }\end{array}$} & \multicolumn{3}{|c|}{$\begin{array}{c}\text { Low stringency protein } \\
\text { identifications }\end{array}$} & \multirow[t]{2}{*}{ High stringency protein identifications } & \multirow[t]{2}{*}{ Protein FDR (\%) } \\
\hline & $R 1$ & $R 2$ & R3 & $R 1$ & $R 2$ & R3 & & \\
\hline Control & 7238 & 6027 & 6099 & 888 & 840 & 821 & 363 & 0.55 \\
\hline ABA & 5269 & 5650 & 5715 & 767 & 1124 & 841 & 310 & ND \\
\hline
\end{tabular}

Abbreviations: FDR, false discovery rate; ND, not detected. R1, R2 and R3 denote replicate 1, replicate 2 and replicate 3 . High-stringency protein indentifications were common to all three replicates. 
Table 2. Annotation of proteins differentially expressed $2 \mathrm{~h}$ after $10 \mu \mathrm{M}$ ABA treatment measured by a label-free method (method 1 )

\begin{tabular}{|c|c|c|c|c|}
\hline Current V1 ID & UniProt ID & Protein annotation & $\log _{2}$ ratio: $A B A / C o n t r o l$ & $\mathrm{P}$ value \\
\hline VIT_10s0042g00200 & F6HIN7 & Thioredoxin $\mathrm{X}$ & -2.43 & $4.86 \mathrm{E}-03$ \\
\hline VITISV_026175 & A5B8D1 & 60S Ribosomal protein L12 family & -1.95 & $2.42 \mathrm{E}-04$ \\
\hline VIT_05s0094g01380 & A5AQ16 & Unknown protein & -1.91 & $8.31 E-03$ \\
\hline VIT_16s0098g01200 & E0CVA1 & NagB/RpiA/CoA transferase-like superfamily protein & -1.63 & $1.49 \mathrm{E}-02$ \\
\hline VIT_11s0052g01710 & D7SQ37 & Xylose isomerase & -1.31 & $2.74 \mathrm{E}-02$ \\
\hline VITISV_041925 & A5AEX6 & alpha/beta-hydrosolase superfamily protein & -1.07 & $2.07 \mathrm{E}-02$ \\
\hline VIT_05s0020g02480 & D7T6P4 & Glutamine_synthetase & -0.84 & $2.19 E-03$ \\
\hline VIT_00s0904g00010 & F6H8B4 & oxygen-evolving enhancer protein 3-2 & -0.78 & $8.20 E-03$ \\
\hline VITISV_016176 & A5BRI2 & Protein kinase superfamily protein & -0.74 & $3.60 \mathrm{E}-02$ \\
\hline VIT_13s0064g01430 & D7T2W3 & RNA-binding (RRM/RBD/RNP motifs) family protein & -0.69 & $3.20 \mathrm{E}-03$ \\
\hline VIT_18s0001g00820 & F6H0D6 & Thioredoxin-like protein CDSP32 & -0.43 & $1.95 E-02$ \\
\hline VIT_19s0014g03850 & A5BX41 & Cytochrome_b6f_complex_ironsulfur_subunit & -0.37 & $1.22 \mathrm{E}-02$ \\
\hline VITISV_017201 & A5AUG8 & Voltage-dependent anion channel 4 & 0.88 & $1.62 \mathrm{E}-02$ \\
\hline VITISV_016936 & A5BYT5 & FRAGILE HISITIDINE TRIAD & 1.51 & $2.89 \mathrm{E}-02$ \\
\hline VIT_05s0020g02880 & F6HDM4 & Glucose1phosphate adenylyltransferase & 2.04 & $3.71 \mathrm{E}-02$ \\
\hline VIT_06s0004g06610 & D7SJX8 & Peptidyl-prolyl cis-trans isomerase & 2.08 & $3.76 \mathrm{E}-02$ \\
\hline VIT_11s0016g03210 & P51615 & NADP-dependent malic enzyme & 2.59 & $5.00 \mathrm{E}-02$ \\
\hline VITISV_008240 & A5BGC9 & 6-phosphogluconate dehydrogenase, decarboxylating & 2.73 & $3.95 \mathrm{E}-03$ \\
\hline VIT_18s0072g01000 & F6GY10 & 2-oxoacid dehydrogenase acyltransferase family protein & 2.79 & $1.95 \mathrm{E}-02$ \\
\hline VIT_14s0030g02180 & F6HTR2 & GDP-mannose 3,5-epimerase 1 & 2.79 & $1.95 \mathrm{E}-02$ \\
\hline VIT_10s0003g03260 & F6HLR2 & Prefoldin 6 & 2.96 & $1.26 \mathrm{E}-03$ \\
\hline VITISV_033715 & A5AZX9 & Triosephosphate isomerase & 3.16 & $1.27 \mathrm{E}-04$ \\
\hline VITISV_033255 & A5AFH5 & Cysteine synthase & 3.96 & $1.16 \mathrm{E}-03$ \\
\hline VIT_13s0019g00260 & Q6XGX7 & Putative oxygen-evolving enhancer protein & 4.00 & $2.30 \mathrm{E}-05$ \\
\hline
\end{tabular}

The phosphoproteins tagged with TMT can be used for further quantification. The localized sites were identified and were reported with Ascore, which calculates the probability of correct phosphorylation site localization based on the presence and intensity of site-determining ions in MS/MS spectra. ${ }^{35}$ From the identified phosphoproteins, 192 non-redundant phosphorylation sites were found with Ascores $\geqslant 13(P<0.05)$ (Supplementary File 7). Identified phosphorylation sites were classified into $77 \%$ pSer, $20 \%$ pThr and 3\% pTyr (Figure 2a). One percent of the proteins had three phosphorylation sites, $7 \%$ of the proteins had two phosphorylation sites and $92 \%$ of the proteins had one phosphorylation site, (Figure 2b). More than $50 \%$ of significant proteins in response to ABA (Table 3) were phosphoproteins. The novel phosphorylation sites with Ascores $\geqslant 13(P<0.05)$ were reported in Supplementary File 7. New phosphorylation sites for many proteins were identified; for example, pectinesterase (F6H777), a potassium efflux antiporter1 (F61616) and an auxin efflux carrier (F6HFI3).

GO analysis for the phosphoproteins found in this study indicated that nucleotide binding is the most over-represented category of molecular function while membrane and transport is the most over-represented categories of the cellular component and biological process, respectively (Figure 3, Supplementary File 8). The plasma membrane was the highest membrane type for phosphoproteins in this study.

The putative motifs surrounding the phosphorylation sites of all phosphopeptides found in this experiment were analyzed with localization probability $\geqslant 95 \%$ and Ascores $\geqslant 13(P \leqslant 0.05)$. Finding of motifs involving phosphorylation events provides valuable information about the specific binding of kinases to substrates. The amino acids around the phosphorylation sites from -6 to +6 were aligned to find the common motifs using WebLogo. The motifs found were $[S-P],[S-D],[R-x-x-S]$ and $[S-x-x-x-x-E]$ for phosphoserine, $[\mathrm{T}-\mathrm{P}]$ for phosphothreonine and $[\mathrm{Y}-\mathrm{G}]$ for phosphotyrosine (Figure 4; Supplementary File 9).

We also observed a similar response of specific phosphoproteins decreased in protein abundance after treatment with $A B A$ to that which has been previously reported in phosphoproteins in Arabidopsis; for example, these included embryonic factor1 (AT2G38280; D7SY29), IQ-domain 32 (AT1G19870; F6H068), seed imbibition 1-like (AT5G40390; D7TWK5) and Tudor/PWWP/MBT protein (AT3G09670; F6HNK4). ${ }^{11,36}$

Furthermore, the same leaf samples were used for all of the transcriptomic, proteomic and phosphoproteomic analysis. Transcriptomic data of ABA-treated leaves was obtained from microarrays, ${ }^{19}$ while proteomic profiles were obtained from TMT and label-free approaches. There were 508 identified proteins found in TMT tagged that were also found in the label-free method (Figure 5a). TMT quantified 787 proteins from 1011 identified proteins, while label-free quantified 360 proteins from 2533 identified proteins (Figure 5b). All three different approaches reported here can identify seven proteins that were found in common (Table 4). Of these seven proteins, just two showed significant effects by ABA, however, their abundance changes were not the same within all three measurement methods. One chlorophyll a/b binding protein (A5BAI4) was increased in protein abundance by ABA based upon the TMT method, but with little or no effect for the label-free and microarray data in the leaves. Interestingly, this protein is phosphorylated. A heat-shock 70 protein, was increased in abundance by $A B A$ in the label-free method, decreased in the TMT method, with little affect on the transcript levels. 


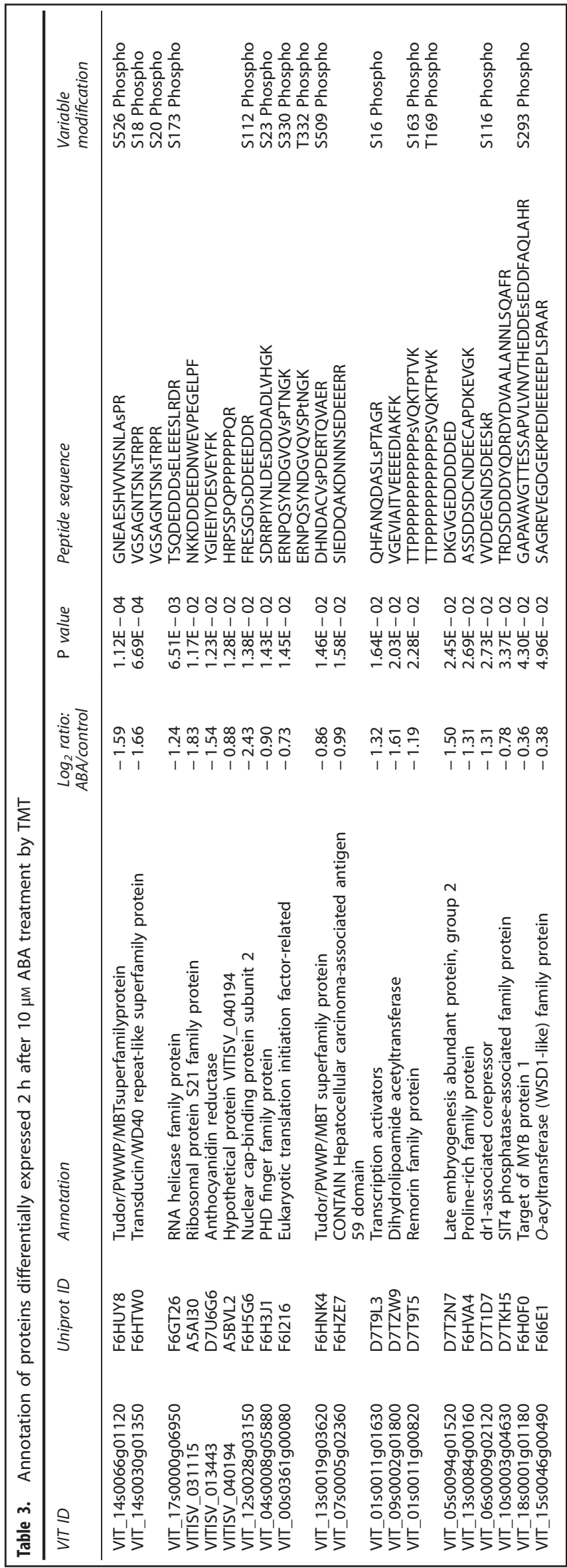

\section{DISCUSSION}

ABA-affected proteins involving plant growth

$A B A$ has multiple physiological effects on plant growth and development. Many of these changes assist the plant to adapt to water deficits, including stomatal closure, photosynthetic protection, antioxidant activities, decreased shoot growth and osmotic adjustment. An increase in endogenous ABA levels is reflective of the degree of water deficit and is normally correlated to growth inhibition. $^{37,38}$ With decreasing soil water potentials, ABA regulates plant growth by inhibiting shoot growth, but promoting root growth. ${ }^{39}$ This is adaptive for the plant resulting in a larger root to shoot ratio, reducing leaf transpiration and increasing water uptake capacity.

Grapevine shoot elongation rate and photosynthesis were inhibited after 4 days of water deficit. However, prior to these physiological changes, there were large changes in protein abundance that were detected. ${ }^{18}$ There was an increase in photosynthetic and antioxidant proteins and a decrease in growth-related proteins for these early changes in response to water deficit. Interestingly, ribosomal proteins involved in protein synthesis were decreased prior to a growth reduction.

The ABA treatment used in this study is likely to represent a concentration of $A B A$ found in leaves exposed to significant water deficit. Our study found a rapid response of proteins to ABA. A number of significant proteins found in our study were involved in organ development. Growth-related proteins were decreased in protein abundance in response to $A B A$, including several ribosomal proteins, an E2 subunit of the mitochondrial pyruvate dehydrogenase complex (D7TZW9), and a Nck-associated protein 1 (D7T9L3) involved in actin remodeling. A mutant of the gene encoding the E2 subunit of the mitochondrial pyruvate dehydrogenase complex in Arabidopsis exhibits retarded growth phenotypes. ${ }^{40}$ Furthermore, ABA inhibits protein synthesis 23,41 and growth. ${ }^{37}$ Leaf growth of maize was inhibited under water deficit, which was related to changes of phosphoproteins involved in cell cycle-related processes. ${ }^{20}$ There were significant changes of phosphoproteins in this study that were involved in growth and organ developmental processes. The changes of abundance of these proteins may result in the plant's acclimation to a drying environment.

Proteins involved in photosynthesis are an important means to control plant growth and development. The inhibition of proteins involved in photosynthesis are correlated with the reduction of shoot elongation of grapevine under water deficit and salinity. ${ }^{42}$ In addition, studies of leaves and fruits treated or affected by ABA showed a decrease in gene expression involved in photosynthesis and a decrease in chlorophyll content. ${ }^{43-45}$ Our study found many significant photosynthetic proteins decreased in protein abundance in response to $A B A$. Overall, our results indicate that early changes in protein abundance in response to $A B A$ involve plant growth and photosynthesis.

Phosphoproteomic analysis reveals novel phosphosites and motifs in the ABA signaling pathway

At the time of writing this manuscript, the Plant Protein Phosphorylation Database (P3DB, http://p3db.org), listed 607 phosphoproteins with 862 phosphosites identified in berries of grapevines ( $V$. vinifera 'Italia $\mathrm{Cv}^{\prime}$ ) using iTRAQ labeling with $\mathrm{TiO}_{2}$-phosphopeptide enrichment. ${ }^{46}$ In comparison, we found 219 phosphoproteins with 192 phosphosites in leaves of $V$. vinifera cv. Cabernet Sauvignon using an IMAC-phosphopeptide enrichment and TMT labeling method. In our study, many novel proteins were modified by phosphorylation. The phosphorylation sites found in the previous works ${ }^{46,47}$ were confirmed in this study, such as the phosphosites for ABCG11 (D7T7C0), RuBisCO (F6GWA8) and serine/threonine-protein kinase (F6HPW0). Our study identified five novel pTyr sites with Ascores $\geqslant 13(P<0.05)$ 
in grapevine proteins. The phosphorylation of tyrosine (Tyr) in plants is less abundant due to the lack of receptor Tyr kinases. ${ }^{48}$ However, in silico analysis of the Arabidopsis genome indicated

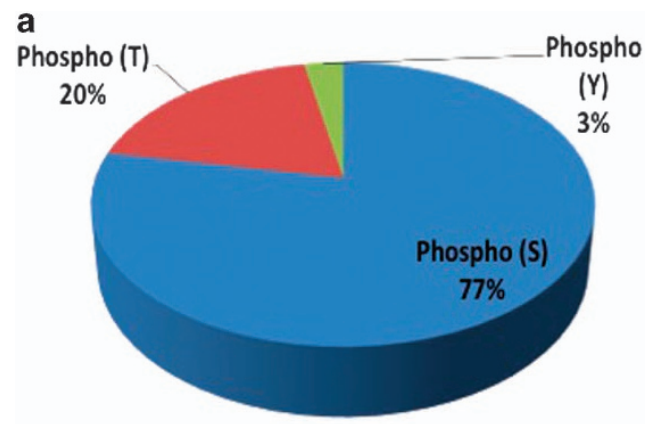

b

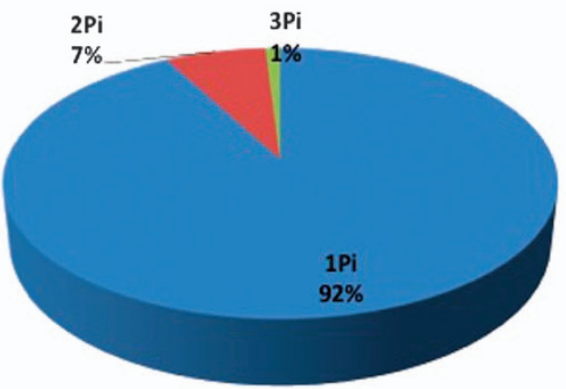

Figure 2. Proportional representation of phosphorylation sites on serine, threonine and tyrosine with Ascores $\geqslant 13(P<0.05)$ found in Cabernet Sauvignon leaf proteins whose roots had been treated with and without $10 \mu \mathrm{m} A B A$ (a) and number of phosphosites, showing that most phosphopeptides were had one phosphosite $(92 \%)$, followed by two phosphosites (7\%) and three phosphosites (1\%) (b). ABA, abscisic acid.

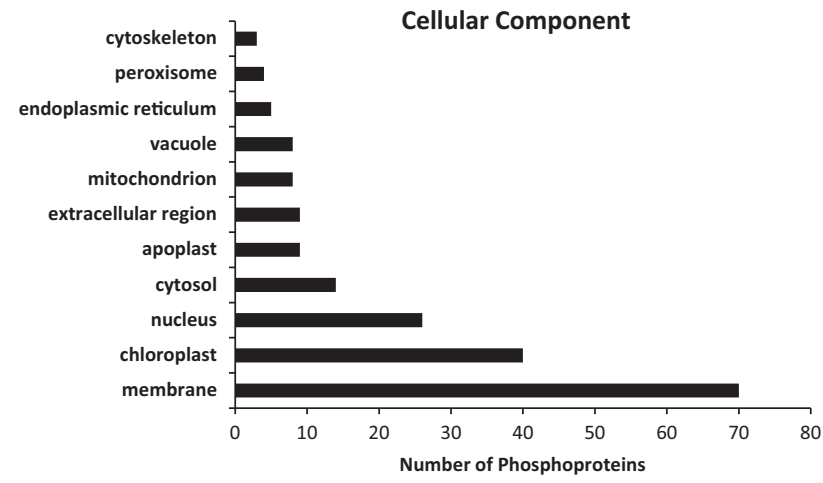

that $\sim 4 \%$ of Arabidopsis kinases are tyrosine-specific kinases, which was close to the amount of pY found in our study. The proportions of phosphorylation sites on serine, threonine and tyrosine found in this study was consistent with the study on other plant species, such as $89.5 \% \mathrm{pS}, 8.9 \% \mathrm{pT}$ and $1.6 \% \mathrm{pY}$ found in rice, and $87.7 \% \mathrm{pS}, 9.9 \% \mathrm{pT}$ and $2.4 \% \mathrm{pY}$ found in Arabidopsis. ${ }^{46,49,50}$

We observed a decrease in protein abundance involved in serine family amino-acid metabolic process in leaves in response to ABA. Serines are commonly phosphorylated by kinases during cell signaling. In plants, the phosphorylated pathway of serine biosynthesis has had an important role in supplying serine to nonphotosynthetic tissues under environmental stresses. ${ }^{51,52}$

The phosphorylation motifs are important to determine the binding of the kinase to its substrate. ${ }^{53}$ The identification of phosphorylation motifs and phosphorylation site localizations are important in understanding many signal transduction pathways. The motifs found in this study have been identified as the possible substrates of SnRK2s. ${ }^{10,36}$ The SnRK2s have been confirmed to phosphorylate $[\mathrm{R}-\mathrm{x}-\mathrm{x}-\mathrm{S}]$ motifs in vitro. ${ }^{14,54}$ We found the phosphorylated $[\mathrm{R}-\mathrm{x}-\mathrm{x}-\mathrm{S}]$ motif of the $\mathrm{ABC}$ transporter $\mathrm{G}$ family member 40 (ABCG40; F6HX69), which decreased in protein abundance in response to $A B A$. An $A B C$ transporter was found to be able to transport $A B A$ from the cytoplasm to the vacuole in order to control the level of $A B A$ in the cytosol. ${ }^{55} A B C G 40$ is responsible for ABA transport into guard cells in Arabidopsis. ${ }^{56}$ The molecular mechanism of how ABA is transported has not yet been fully elucidated. It is possible that phosphorylation might be involved in this $A B A$ transport mechanism.

Normally, membranes are the first sites of signaling to occur in response to stresses. In this context, many phosphoproteins found in this study are membrane proteins. It has been found that transport systems tend to be phosphorylated, ${ }^{57,58}$ which corresponds to our finding that transport is a major biological process of phosphoproteins. Altogether, these results indicate that $A B A$

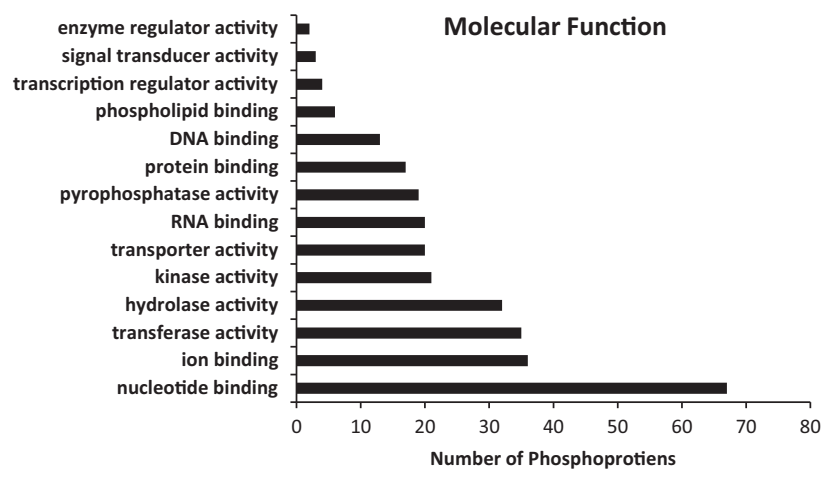

Biological Process

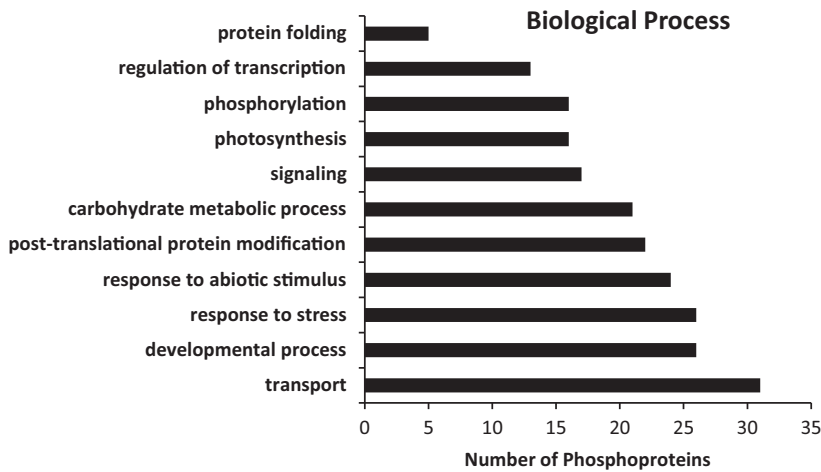

Figure 3. Functional categories of 219 phosphoproteins identified by TMT-LC-MS/MS were analyzed with the Cytoscape (3.2.0) and BinGo plugin (3.0.3). LC-MS, liquid chromatography tandem mass spectrometry; TMT, tandem mass tags. 


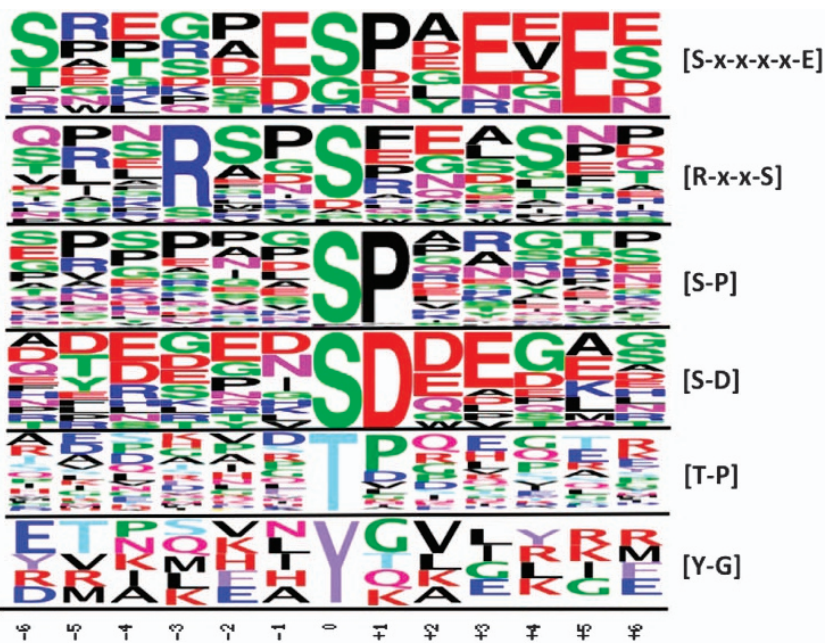

Figure 4. Sequence logos of all phosphorylation sites with Ascores $\geqslant$ $13(P \leqslant 0.05)$. The frequency of each residue present in each data set is proportional to its height. The phosphorylation site is 0 on the $x$ axis flanking by 12 amino-acid residues (at position -6 to +6 ). Extracted motifs were shown on the right.

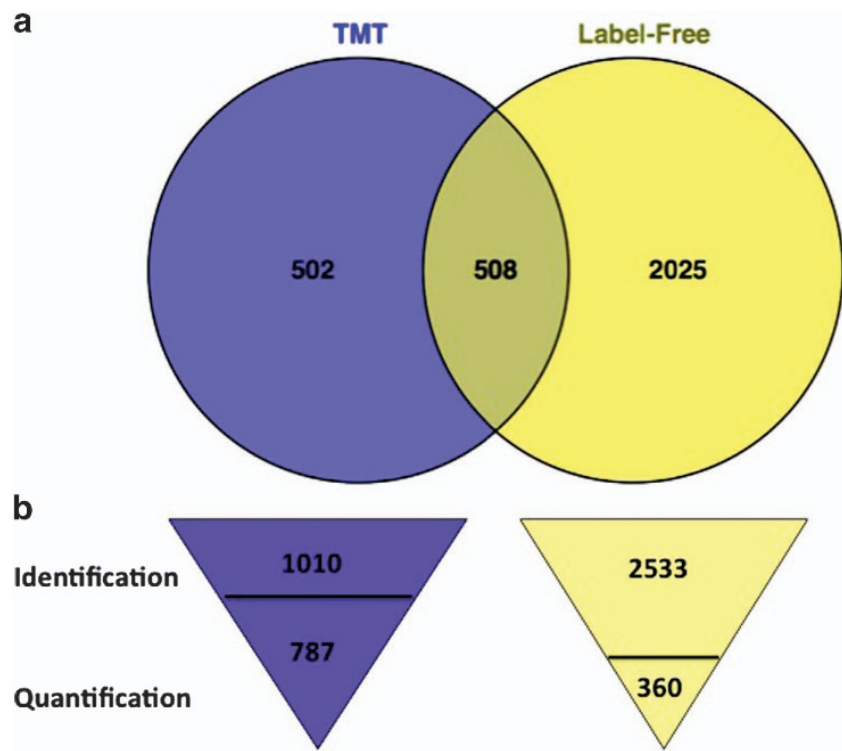

Figure 5. The overlap of proteins found in TMT and label-free method (a), and number of protein identification and quantification from TMT and label-free method (b).

\begin{tabular}{|c|c|c|c|c|c|c|c|}
\hline \multirow[t]{2}{*}{ Current V1 ID } & \multirow[t]{2}{*}{ UniProt ID } & \multirow[t]{2}{*}{ Annotation } & \multicolumn{2}{|c|}{ Microarray } & \multirow{2}{*}{$\frac{\text { Label-free }}{\log _{2} L A / L C}$} & \multirow{2}{*}{$\frac{T M T}{\log _{2} L A / L C}$} & \multirow[t]{2}{*}{ Phosphorylated } \\
\hline & & & $\log _{2} L A / L C$ & $\log _{2} R A / R C$ & & & \\
\hline VIT_10s0003g02890 & A5BAI4 & Chlorophyll a/b binding protein 3 & -0.045 & 2.133 & 0.188 & 0.934 & Yes \\
\hline VIT_06s0061g00270 & F6GWA8 & Chaperonin 60 subunit alpha 1, chloroplastic & 0.062 & 1.431 & 0.321 & -0.363 & Yes \\
\hline VIT_14s0060g00820 & A5AIE0 & $\begin{array}{l}\text { Chloroplast stem-loop binding protein of } 41 \mathrm{kDa} \\
\mathrm{b} \text {, chloroplastic }\end{array}$ & 0.036 & 1.284 & 0.291 & -0.561 & \\
\hline VIT_06s0004g00240 & D7SLM9 & Chaperonin 60 subunit beta 3 , chloroplastic & -0.044 & 1.050 & 0.015 & -0.216 & \\
\hline VIT_15s0024g00040 & F61519 & Photosystem I light harvesting complex gene 3 & 0.090 & 1.705 & -0.385 & 0.234 & \\
\hline
\end{tabular}

may be involved in the regulation of membrane transport systems in grapevine leaves via a protein phosphorylation process. ${ }^{58}$

Analysis of omics in response to $A B A$

Label-free and labeling approaches have been found to be equally capable of reliably and accurately quantifying protein abundance levels. ${ }^{59,60}$ Our results confirmed the previous finding ${ }^{61}$ that compared label-free and an isobaric chemical labeling method. Combining label-free with the labeling approach provides a more complete picture for a proteomic study.

On the basis of our results of transcriptomic, proteomic and phosphoproteomic analyses, genes that significantly increased in transcript abundance in response to ABA in the roots, also had their protein abundance changed in the leaves. There might be a signal from the roots causing a change in protein abundance in the leaves. ABA has been proposed as a root-to-shoot signal during drought stress. $^{62,63}$ Our work presented here demonstrated the changing of proteins in the leaves resulting from ABA-treated roots. The protein amino-acid phosphorylation process was a significantly overrepresented GO category of DEGs in response to ABA in roots, whereas the organ development process was a significantly overrepresented GO category for significant proteins in leaves. More than $50 \%$ of significant proteins in response to $A B A$ were phosphoproteins.
This finding indicated that there is a communication between roots and leaves in response to $A B A$, which may involve phosphorylation.

\section{CONCLUSIONS}

Utilization of proteomics and phosphoproteomics has provided the data that has lead to deeper understanding of ABA responses in both proteins and phosphorylation of those proteins. With the short-term treatment of $A B A$, we discovered rapid and significant changes in protein abundance in the leaves of roots treated with exogenous $A B A$. This finding indicated that there is rapid communication between roots and leaves when responding to $A B A$. ABA decreased the abundance of growth-related and photosynthetic proteins, probably in an effort to reduce leaf area and water loss. Many potential target proteins and phosphoproteins for ABA signaling were identified. Phosphoproteins found in this study were membrane proteins involved in transport and nucleotide binding. These transport proteins may be involved in plant growth and adaptation to water deficits.

\section{CONFLICT OF INTEREST}

The authors declare no conflict of interest. 


\section{ACKNOWLEDGEMENTS}

This work was funded by the College of Agriculture and Natural Resources at the University of Nevada, Reno with a Nevada Agriculture Experimental Station MultiState Hatch Grant (Grant \# NEV00345).

\section{REFERENCES}

1 Cramer GR. Abiotic stress \& plant responses from the whole vine to the genes. Aust J Grape Wine Res 2010; 16: 86-93.

2 Hannah L, Roehrdanz PR, Ikegami M, Shepard AV, Shaw MR, Tabor G et al. Climate change, wine, and conservation. Proc Natl Acad Sci USA 2013; 110: 6907-6912.

3 Deis L, Cavagnaro B, Bottini R, Wuilloud R, Fernanda Silva M. Water deficit and exogenous $A B A$ significantly affect grape and wine phenolic composition under in field and in-vitro conditions. Plant Growth Regul 2011; 65: 11-21.

4 Deluc LG, Quilici DR, Decendit A, Grimplet J, Wheatley MD, Schlauch KA et al. Water deficit alters differentially metabolic pathways affecting important flavor and quality traits in grape berries of Cabernet Sauvignon and Chardonnay. BMC Genomics 2009; 10: 212.

5 Grimplet J, Deluc LG, Tillett RL, Wheatley MD, Schlauch KA, Cramer GR et al. Tissue-specific mRNA expression profiling in grape berry tissues. BMC Genomics 2007; 8: 187.

6 Van Leeuwen C, Tregoat O, Chone X, Bois B, Pernet D, Gaudillere JP. Vine water status is a key factor in grape ripening and vintage quality for red bordeaux wine. how can it be assessed for vineyard management purposes? J Int Sci Vigne Vin 2009; 43: 121-134.

7 Kermode AR. Role of abscisic acid in seed dormancy. J Plant Growth Regul 2005 24: 319-344.

8 Nambara E, Marion-Poll A. Abscisic acid biosynthesis and catabolism. Annu Rev Plant Biol 2005; 56: 165-185.

9 Wang P, Du Y, Hou YJ, Zhao Y, Hsu CC, Yuan F et al. Nitric oxide negatively regulates abscisic acid signaling in guard cells by S-nitrosylation of OST1. Proc Nat/ Acad Sci USA 2015; 112: 613-618.

10 Umezawa T, Sugiyama N, Takahashi F, Anderson JC, Ishihama Y, Peck SC et al. Genetics and phosphoproteomics reveal a protein phosphorylation network in the abscisic acid signaling pathway in Arabidopsis thaliana. Sci Signal 2013; 6: rs8.

11 Kline KG, Barrett-Wilt GA, Sussman MR. In planta changes in protein phosphorylation induced by the plant hormone abscisic acid. Proc Natl Acad Sci USA 2010; 107: 15986-15991.

12 Umezawa T, Nakashima K, Miyakawa T, Kuromori T, Tanokura M, Shinozaki K et al. Molecular basis of the core regulatory network in aba responses: sensing, signaling and transport. Plant Cell Physiol 2010; 51: 1821-1839.

13 Lumba S, Toh S, Handfield LF, Swan M, Liu R, Youn JY et al. A mesoscale abscisic acid hormone interactome reveals a dynamic signaling landscape in Arabidopsis. Dev Cell 2014; 29: 360-372.

14 Kobayashi Y, Murata M, Minami H, Yamamoto S, Kagaya Y, Hobo T et al. Abscisic acid-activated SNRK2 protein kinases function in the gene-regulation pathway of $A B A$ signal transduction by phosphorylating $A B A$ response element-binding factors. Plant J 2005; 44: 939-949.

15 Lee SC, Lan W, Buchanan BB, Luan S. A protein kinase-phosphatase pair interacts with an ion channel to regulate ABA signaling in plant guard cells. Proc Natl Acad Sci USA 2009; 106: 21419-21424.

16 Cramer GR, Urano K, Delrot S, Pezzotti M, Shinozaki K. Effects of abiotic stress on plants: a systems biology perspective. BMC Plant Biol 2011; 11: 163.

17 Ghan R, Van Sluyter SC, Hochberg U, Degu A, Hopper DW, Tillet RL et al. Five omic technologies are concordant in differentiating the biochemical characteristics of the berries of five grapevine (Vitis vinifera L.) cultivars. BMC Genomics 2015; 16: 946.

18 Cramer GR, Van Sluyter SC, Hopper DW, Pascovici D, Keighley T, Haynes PA. Proteomic analysis indicates massive changes in metabolism prior to the inhibition of growth and photosynthesis of grapevine (Vitis vinifera L.) in response to water deficit. BMC Plant Biol 2013; 13: 49.

19 Rattanakon S, Ghan R, Gambetta G, Deluc L, Schlauch KA, Cramer GR. Transcriptomic analysis reveals that abscisic acid signaling differs in different organs of grapevine (Vitis vinifera L.). BMC Plant Biol 2015; 16: 72.

20 Bonhomme L, Valot BA, Tardieu Fß, Zivy M. Phosphoproteome dynamics upon changes in plant water status reveal early events associated with rapid growth adjustment in maize leaves. Mol Cell Proteomics 2012; 11: 957-972.

21 de la Fuente van Bentem S, Anrather D, Roitinger E, Djamei A, Hufnagl T, Barta A et al. Phosphoproteomics reveals extensive in vivo phosphorylation of Arabidopsis proteins involved in RNA metabolism. Nucleic Acids Res 2006; 34: 3267-3278.

22 Grimsrud PA, den OD, Wenger CD, Swaney DL, Schwartz D, Sussman MR et al. Large-scale phosphoprotein analysis in Medicago truncatula roots provides insight into in vivo kinase activity in legumes. Plant Physiol 2010; 152: 19-28.
$23 \mathrm{Hu}$ X, Li N, Wu L, Li C, Li C, Zhang L et al. Quantitative iTRAQ-based proteomic analysis of phosphoproteins and ABA-regulated phosphoproteins in maize leaves under osmotic stress. Sci Rep 2015; 5: 15626.

24 van Wijk KJ, Friso G, Walther D, Schulze WX. Meta-Analysis of Arabidopsis thaliana Phospho-Proteomics Data Reveals Compartmentalization of Phosphorylation Motifs. The Plant Cell 2014; 26: 2367-2389.

25 Gibeaut DM, Hulett J, Cramer GR, Seemann JR. Maximal biomass of Arabidopsis thaliana using a simple, low-maintenance hydroponic method and favorable environmental conditions. Plant Physiology 1997; 115: 317-319.

26 George IS, Pascovici D, Mirzaei M, Haynes PA. Quantitative proteomic analysis of Cabernet Sauvignon grape cells exposed to thermal stresses reveals alterations in sugar and phenylpropanoid metabolism. Proteomics 2015; 15: 3048-3060.

27 Gammulla CG, Pascovici D, Atwell BJ, Haynes PA. Differential metabolic response of cultured rice (Oryza sativa) cells exposed to high- and low-temperature stress. Proteomics 2010; 10: 3001-3019.

28 Neilson KA, Keighley T, Pascovici D, Cooke B, Haynes PA. Label-free quantitative shotgun proteomics using normalized spectral abundance factors. Methods $\mathrm{Mol}$ Biol 2013; 1002: 205-222.

29 Keller A, Nesvizhskii Al, Kolker E, Aebersold R. Empirical statistical model to estimate the accuracy of peptide identifications made by MS/MS and database search. Anal Chem 2002; 74: 5383-5392.

30 Dayon L, Hainard A, Licker V, Turck N, Kuhn K, Hochstrasser DF et al. Relative quantification of proteins in human cerebrospinal fluids by MS/MS using 6-plex isobaric tags. Anal Chem 2008; 80: 2921-2931.

31 Schwartz D, Gygi SP. An iterative statistical approach to the identification of protein phosphorylation motifs from large-scale data sets. Nat Biotechnol 2005; 23: $1391-1398$.

32 Crooks GE, Hon G, Chandonia JM, Brenner SE. WebLogo: a sequence logo generator. Genome Res 2004; 14: 1188-1190.

33 Maere S, Heymans K, Kuiper M. BiNGO: a Cytoscape plugin to assess overrepresentation of gene ontology categories in biological networks. Bioinformatics 2005; 21: 3448-3449.

34 Shannon P, Markiel A, Ozier O, Baliga NS, Wang JT, Ramage D et al. Cytoscape: a software environment for integrated models of biomolecular interaction networks. Genome Res 2003; 13: 2498-2504.

35 Beausoleil SA, Villen J, Gerber SA, Rush J, Gygi SP. A probability-based approach for high-throughput protein phosphorylation analysis and site localization. Nat Biotechnol 2006; 24: 1285-1292.

36 Wang P, Xue L, Batelli G, Lee S, Hou YJ, Van Oosten MJ et al. Quantitative phosphoproteomics identifies SnRK2 protein kinase substrates and reveals the effectors of abscisic acid action. Proc Natl Acad Sci USA 2013; 110: 11205-11210.

37 Munns R, Cramer GR. Is coordination of leaf and root growth mediated by abscisic acid? Opinion. Plant Soil 1996; 185: 33-49.

38 Sharp RE, LeNoble ME, Else MA, Thorne ET, Gherardi F. Endogenous ABA maintains shoot growth in tomato independently of effects on plant water balance: evidence for an interaction with ethylene. J Exp Bot 2000; 51: 1575-1584.

39 Saab IN, Sharp RE, Pritchard J, Voetberg GS. Increased endogenous abscisic acid maintains primary root growth and inhibits shoot growth of maize seedlings at low water potentials. Plant Physiol 1990; 93: 1329-1336.

40 Song L, Liu D. Mutations in the three Arabidopsis genes that encode the E2 subunit of the mitochondrial pyruvate dehydrogenase complex differentially affect enzymatic activity and plant growth. Plant Cell Rep 2015; 34: 1919-1926.

41 Paranjothy K, Wareing PF. The effects of abscisic acid, kinetin and 5-fluorouracil on ribonucleic acid and protein synthesis in senescing radish leaf disks. Planta 1971; 99: 112-119.

42 Vincent D, Ergul A, Bohlman MC, Tattersall EA, Tillett RL, Wheatley MD et al. Proteomic analysis reveals differences between Vitis vinifera L. cv. Chardonnay and cv. Cabernet Sauvignon and their responses to water deficit and salinity. J Exp Bot 2007; 58: 1873-1892.

43 Koyama K, Sadamatsu K, Goto-Yamamoto N. Abscisic acid stimulated ripening and gene expression in berry skins of the Cabernet Sauvignon grape. Funct Integr Genomics 2010; 10: 367-381.

44 Mou W, Li D, Luo Z, Mao L, Ying T. Transcriptomic Analysis Reveals Possible Influences of ABA on Secondary Metabolism of Pigments, Flavonoids and Antioxidants in Tomato Fruit during Ripening. PLOS ONE 2015; 10: e0129598.

45 Liang C, Wang Y, Zhu Y, Tang J, Hu B, Liu L et al. OsNAP connects abscisic acid and leaf senescence by fine-tuning abscisic acid biosynthesis and directly targeting senescence-associated genes in rice. Proc Natl Acad Sci USA 2014; 111: 10013-10018.

46 Melo-Braga MN, Verano-Braga T, Leon IR, Antonacci D, Nogueira FC, Thelen J et al. Modulation of protein phosphorylation, $\mathrm{N}$-glycosylation and Lys-acetylation in grape (Vitis vinifera) mesocarp and exocarp owing to Lobesia botrana infection. Mol Cell Proteomics 2012; 11: 945-956. 
47 Yao Q, Ge H, Wu S, Zhang N, Chen W, Xu C et al. P(3)DB 3.0: From plant phosphorylation sites to protein networks. Nucleic Acids Res 2014; 42: D1206-D1213.

48 Luan S. Tyrosine phosphorylation in plant cell signaling. Proc Natl Acad Sci USA 2002; 99: 11567-11569.

49 Nakagami H, Sugiyama N, Mochida K, Daudi A, Yoshida Y, Toyoda T et al. Largescale comparative phosphoproteomics identifies conserved phosphorylation sites in plants. Plant Physiol 2010; 153: 1161-1174.

50 Sugiyama N, Nakagami H, Mochida K, Daudi A, Tomita M, Shirasu K et al. Largescale phosphorylation mapping reveals the extent of tyrosine phosphorylation in Arabidopsis. Mol Syst Biol 2008; 4: 193.

$51 \mathrm{Ho} C L$, Saito K. Molecular biology of the plastidic phosphorylated serine biosynthetic pathway in Arabidopsis thaliana. Amino Acids 2001; 20: 243-259.

$52 \mathrm{Ho}$ CL, Noji M, Saito M, Saito K. Regulation of serine biosynthesis in Arabidopsis. Crucial role of plastidic 3-phosphoglycerate dehydrogenase in nonphotosynthetic tissues. J Biol Chem 1999; 274: 397-402.

53 Kettenbach AN, Wang T, Faherty BK, Madden DR, Knapp S, Bailey-Kellogg C et al. Rapid determination of multiple linear kinase substrate motifs by mass spectrometry. Chem Biol 2012; 19: 608-618.

54 Furihata T, Maruyama K, Fujita Y, Umezawa T, Yoshida R, Shinozaki K et al. Abscisic acid-dependent multisite phosphorylation regulates the activity of a transcription activator AREB1. Proc Natl Acad Sci USA 2006; 103: 1988-1993.

55 Burla B, Pfrunder S, Nagy R, Francisco RM, Lee Y, Martinoia E. Vacuolar transport of abscisic acid glucosyl ester is mediated by ATP-binding cassette and protonantiport mechanisms in Arabidopsis. Plant Physiol 2013; 163: 1446-1458.

56 Kang J, Hwang JU, Lee M, Kim YY, Assmann SM, Martinoia E et al. PDR-type ABC transporter mediates cellular uptake of the phytohormone abscisic acid. Proc Nat Acad Sci USA 2010; 107: 2355-2360.

57 Nuhse TS, Stensballe A, Jensen ON, Peck SC. Phosphoproteomics of the Arabidopsis plasma membrane and a new phosphorylation site database. Plant Cell 2004; 16: 2394-2405.
58 Osakabe Y, Yamaguchi-Shinozaki K, Shinozaki K, Tran LS. ABA control of plant macroelement membrane transport systems in response to water deficit and high salinity. New Phytol 2014; 202: 35-49.

59 Neilson KA, Ali NA, Muralidharan S, Mirzaei M, Mariani M, Assadourian G et al. Less label, more free: approaches in label-free quantitative mass spectrometry. Proteomics 2011; 11: 535-553.

60 Turck CW, Falick AM, Kowalak JA, Lane WS, Lilley KS, Phinney BS et al. The Association of Biomolecular Resource Facilities Proteomics Research Group 2006 study: relative protein quantitation. Mol Cell Proteomics 2007; 6: 1291-1298.

61 Li Z, Adams RM, Chourey K, Hurst GB, Hettich RL, Pan C. Systematic comparison of label-free, metabolic labeling, and isobaric chemical labeling for quantitative proteomics on LTQ Orbitrap Velos. J Proteome Res 2012; 11: 1582-1590.

62 Davies W, Kudoyarova G, Hartung W, Long-distance ABA. Signaling and Its relation to other signaling pathways in the detection of soil drying and the mediation of the Plant,Äôs response to drought. J Plant Growth Regul 2005; 24: 285-295.

63 Sauter A, Davies WJ, Hartung W. The long-distance abscisic acid signal in the droughted plant: the fate of the hormone on its way from root to shoot. J Exp Bot 2001; 52: 1991-1997.

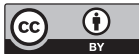

This work is licensed under a Creative Commons Attribution 4.0 International License. The images or other third party material in this article are included in the article's Creative Commons license, unless indicated otherwise in the credit line; if the material is not included under the Creative Commons license, users will need to obtain permission from the license holder to reproduce the material. To view a copy of this license, visit http://creativecommons.org/licenses/ by/4.0/

(c) The Author(s) 2016

Supplementary Information for this article can be found on the Horticulture Research website (http://www.nature.com/hortres) 